\title{
The role of inflammation in stress cardiomyopathy
}

Article Type: Invited Review

Corresponding Author:

Dana Dawson, DM, FRCP, D.Phil, FESC

Professor of Cardiovascular Medicine and Consultant Cardiologist

Corresponding Author's Institution:

School of Medicine, Medical Sciences \& Nutrition, University of Aberdeen,

Foresterhill, Aberdeen, Scotland, United Kingdom

Corresponding Author's E-mail Address:

dana.dawson@abdn.ac.uk 
Abstract:

Stress cardiomyopathy (SC) is an increasingly recognised form of acute heart failure, which has been linked to a wide variety of emotional and physical triggers. The pathophysiological mechanisms of the disease remain incompletely understood, however, inflammation has been recently shown to play a pivotal role. This review summarises the most notable findings of myocardial inflammation, demonstrated from biopsies and cardiac magnetic resonance imaging in humans. In the acute stage macrophage infiltration appears to represent the substrate for myocardial oedema, together defining the local myocardial inflammation. This appears to evolve into a low grade systemic chronic inflammation which could explain the protracted clinical course of these patients and raises hope for finding a specific SC cardiac biomarker as well as a therapeutic breakthrough. As a parallel to the human findings the review covers some of the emerging mechanistic insights from experimental models, which, albeit not proven in the human condition, highlight the possible importance in pursuing distinct paths of investigation such as the betareceptor signaling, aberrations of nitric oxide generation and signaling and the contribution of the vascular endothelium/permeability to oedema and inflammation during the acute stage. 


\section{INTRODUCTION}

Acute stress cardiomyopathy (SC), also known as takotsubo cardiomyopathy, is an acute heart failure syndrome, first noticed in 1990 by Sato in Japan. [1] The eponym of "takotsubo" refers to an octopus trap used by Japanese fishermen and alludes to the typical shape of the left ventricle in this syndrome, consisting of a dyskinetic apex and relatively hyperkinetic base. However, since the first description of the disease more types of SC have been identified: basal, mid-ventricular and focal subtypes. SC has initially been diagnosed more commonly in middle-aged and elderly women, thought to represent $\sim 90 \%$ of the cases, although the condition is increasingly seen in women of all ages as well as men. [2] The hallmark of this cardiomyopathy is being precipitated by a stressor event, either of an emotional or physical nature (or sometimes combined). Clinically, it presents similarly to acute coronary syndromes with chest pain, breathlessness, ECG changes (ST- elevation, T wave changes) and only mildly-moderately elevated cardiac biomarkers. However, unlike myocardial infarction, coronary angiography shows no acute coronary thrombosis, making this the most notable contributor in the differential diagnosis (Mayo Clinic; European Cardiology Society criteria) [3, 4]. Approximately $2-3 \%$ of the initially suspected acute coronary syndromes patients are in fact sufferers of SC. [5] Cardiac imaging shows that the left ventricular dysfunction usually extends beyond the territory of a single coronary artery. While the left ventricular ejection fraction and regional dyskinesia recover within days to weeks, other parameters such as echocardiography derived global longitudinal strain or apical twist have shown to remain decreased over months after the acute episode. [6] This contradicts the initial view according to which SC is a transient and fully reversible disease. Although an acute, in-hospital 
mortality rate of $4-5 \%$ has been recognised, it is relatively recent that registry-derived data reported relatively high long-term rates of major adverse cardiac and cerebrovascular events, which are comparable to those seen after acute coronary syndromes. [7]

It follows therefore, that the pathological processes occurring within the left ventricular myocardium are of significant importance and directly contribute towards this guarded prognosis. This review will describe the current knowledge on the myocardial inflammatory processes in SC and analyses how these may contribute to the recovery and outcomes in the aftermath of an acute episode. Where possible, these will be correlated with currently available data from myocardial biopsies, imaging (cardiac magnetic resonance) and systemic inflammatory processes. Finally, reference to some of the most relevant experimental work will be made, to highlight possible avenues of further investigation with the caveat that the experimental models of SC are simplistic compared to the much more complex human syndrome.

\section{CARDIAC IMAGING}

Whilst echocardiography remains the cornerstone of cardiac imaging in routine clinical care, cardiac magnetic resonance (CMR) imaging has specific application in $\mathrm{SC}$, as it is additive to the information already obtained [8].

Firstly, CMR is able to rule out a myocardial infarction (MI) in SC. Whilst rare occurrences of fibrosis have been reported, the characteristic pattern of myocardial fibrosis that is diagnostic of a MI is absent in SC: the dense myocardial fibrosis seen in $\mathrm{MI}$ seen as late gadolinium enhancement and located subendocardially or transmurally is absent in SC [9]. 
Secondly, CMR is better able to offer an appreciation of the extent of the wall motion abnormality, being also the gold standard for right ventricular imaging. As a result, cardiac MRIs have also unexpectedly revealed a substantial right ventricular involvement, resulting in biventricular ballooning. Approximately $40-50 \%$ of cases have right ventricular dysfunction concomitantly present, which was associated with a longer hospitalization period and increased rate of complications. [10] Moreover, cine CMR sequences are used to underline the overall contraction abnormalities, consisting usually of anterior, antero- lateral and antero-septal walls dyskinesia, alongside a hyperkinetic base. The atypical forms are also well identified on CMR: basal (inverted), mid-ventricular and especially the focal one.

Further on, ${ }^{31} \mathrm{P}$-magnetic resonance spectroscopy has revealed a major impairment in the energetic status both acutely and after period of 3-5 months (decreased PCr/ $/ \mathcal{L}$-ATP ratio). [8] The cardiomyocyte adaptability to the decreased energy production is reflected in the increased expression of survival pathways seen from myocardial biopsies: upregulation of Phosphoinositide 3-kinases (PI3K) and downregulation of Phosphatase and tensin homolog (PTEN; PI3K antagonist). Antiapoptotic pathways (B-cell lymphoma 2-associated X protein (Bax) /B- cell lymphoma 2 (Bcl-2) ratio) also appear activated. These support a mechanism of protection against myocardial apoptosis and necrosis. [11]

Fourthly, CMR is the only imaging modality that is able to provide a qualitative or quantitative appreciation of the myocardial oedema seen in acute SC, as well as to examine its resolution time course during follow-up. T2-weighted images have been the initial investigation method in vivo for detecting acute myocardial oedema: multiple studies have now agreed on this one common finding in acute SC patients (Table 1). T1 or T2 mapping has developed as a more robust, quantifiable method, 
being less sensitive to motion artefacts. Myocardial oedema is most likely a result of increased vascular and cell membrane permeability. Thus, any oedematous tissue corresponds to an increase in tissue water content, which is consequently reflected in the prolonged $\mathrm{T} 1$ and $\mathrm{T} 2$ times. The native $\mathrm{T} 1$ values have been found to be consistently increased in acute SC patients in the entire left ventricular myocardium compared to controls - but particularly in the apical and mid-ventricular regions, which are most commonly affected. [12] Unlike myocardial infarction, the oedema is not overlying a single vascular territory and it appears to affect the whole myocardium, beyond the segments affected by various degrees of dysfunction on cine imaging. The characteristic spontaneous recovery of the left ventricular ejection fraction has misled the scientific community into predicting a fast and benign resolution of the disease. However, subsequent studies showed that myocardial oedema persists for several months. Both T2-weighted semi-qualitative sequences as well as parametric imaging such as previously mentioned T1 mapping demonstrated unresolved left ventricular myocardial oedema compared to controls 34 months after the acute episode. [13] Furthermore, myocardial oedema appears present and also persist for the same time in the right ventricular myocardium. [14] A recent study with follow-up extended to 5 months showed for the first time that it is only at this stage that the oedema begins to dissipate in the myocardium. [8]

Supporting information has been provided from myocardial biopsy studies which demonstrated that the myocardial oedema may subtend an underlying inflammatory process. Analysis of the myocardial tissue has been performed both acutely and in the recovery period. A typical finding from light microscopy in the acute phase revealed hypertrophied myocytes and glycogen present in the cytoplasm. A net reduction in the glycogen content was subsequently observed a few months into 
recovery. Moreover, localized contraction band necrosis, as well as widening of interstitial spaces were also observed in myocardial biopsies. Myocardial electron microscopy reveals numerous vacuoles with debris and myelin bodies in the acute phase. Sporadically, mis-shaped or mis-sized mitochondria have also been noted. Immunohistochemical staining revealed infiltration of neutrophils and macrophages, even T cells - the inflammatory response persisting up to 3 months. [15] Moreover, immunohistological studies have revealed an increased presence of 3-nitrotyrosine (3-NT) and poly-ADP ribose (PAR) in the diseased myocardium from deceased SC patients. These have been linked to nitrosative stress and could be a possible explanation for the energy depletion and negative inotropy representative in the pathology of the cardiomyopathy. [16] While the biopsies have the potential to highlight specific features of SC, setting key differences from other forms of acute heart failure (e.g. in myocarditis lymphocytic infiltrate predominates) became the next challenge.

However, human post-mortem biopsies from patients who demised shortly after the onset (first 5 days) demonstrated clusters of CD68+ cells, suggesting that aggregates of macrophages accumulate in these patients' hearts. Although these data were restricted to unfortunate, fatal cases, the insight provided was substantial as such examinations provided information from a much larger myocardial territory relative to that arising from a biopsy. [17] Although there are limitations in extrapolating pathophysiology similarities form an animal model of SC cardiomyopathy, further insight has been gained from a time course assessment of the experimental model. An experimental Takotsubo rodent model suggests that the catecholamine-induced SC-like cardiac dysfunction culminates with the M1 polarised macrophages infiltration (proinflammatory). This subtype is specialised in extra- 
cellular matrix degradation and release of proinflammatory mediators. In contrast to Ml cases, a transition towards M2 macrophages (anti-inflammatory) is not apparent. [17]. This was also observed in the few post-mortem human myocardial sections examined, however, it raised a further question: is this myocardial infiltrate present only in the patients who died or is it a general characteristic of the disease in all takotsubo patients?

To this end, ultra-small superparamagnetic particles of iron-oxide (USPIO) enhanced CMR has recapitulated, non-invasively, the presence of macrophage infiltration within the myocardial tissue of all takotsubo patients. [8] USPIO uptake has been used in this case to highlight the presence of activated/phagocytic macrophages in the myocardium, serving as an imaging biomarker of inflammation. Thus, USPIO uptake irrefutably proves a macrophage-mediated intracardiac inflammation in all acute SC cases, not just in a few post-mortem examples which could have represented extreme situations that resulted in fatality. The macrophage infiltrate is present acutely in both ballooning and non-ballooning LV segments and becomes attenuated at 5 months after acute presentation. The USPIO uptake does not only explain for the first time at a large scale the substrate of the global myocardial oedema, but it also provides a contrast to other types of heart failure presentations mediated by inflammation, where other immune cells predominate (such as acute myocarditis).

\section{INFLAMMATORY PROCESS}

The presence of global myocardial oedema alongside the macrophage infiltrates further suggested that systemic inflammation might play a pivotal role in the 
pathology of the disease. Acute phase proteins (specifically C - reactive protein) and stretch markers ( $\mathrm{N}$ terminal-prohormone brain natriuretic peptide - NT-pro BNP) have been first tested given the myocardial oedema in SC. They were both found to be considerably elevated, supporting the notion that systemic inflammation is involved in the pathological process. Interestingly, considering that much less myocardial injury/necrosis occurs in SC, CRP levels are comparable to ST-elevation myocardial infraction (STEMI) patients and showed an upward trend after admission. This suggests that this might be a distinct phenomenon and does not necessarily follow the patterns of cellular injury and death. Compared to ST-elevation MI, the cardiac troponins and Creatine Kinase-Muscle Bound isotype are consistently lower, yet BNP levels were considerably elevated, by three to four-fold. [18] These findings have directed researchers into proposing a unique biomarker as the NTproBNP/cardiac troponin T ratio. This ratio distinguishes SC from STEMI with a $91 \%$ sensitivity and 95\% specificity. [19] Elevated CRP and BNP also bear prognostic importance: cardiac complications and even in-hospital mortality were associated with increased serum presence of both above-mentioned proteins. It is therefore likely that elevated NT-proBNP and BNP levels in SC have a dual significance, both inflammatory and myocardial stretch being causative, but the former probably having a predominant role. Supporting the inflammatory role is the fact that the concentration of BNP/ NT-proBNP remains borderline elevated at a 3-month followup. In addition, strong correlations between NT-proBNP, normetanephrine concentration and the severity of the disease (left ventricular ejection fraction) have been demonstrated. [20]

It has been postulated that BNP could have a protective effect against reactive oxygen species damage, improving the hemodynamic imbalance by acting as a 
second messenger in the cGMP pathway. The outcome of BNP release has been linked to limiting intra-cardiac inflammation by inhibiting the neutrophil burst and the subsequent reactive oxygen species formation, while at the same time avoiding the risks of NO release (mainly nitrosative stress). Nonetheless, there is not enough supporting evidence for a possible protective mechanistic role for BNP.

NO has the capacity to bind to the superoxide anion (O2-) leading to the formation of peroxynitrite anion (ONOO-). As a result, redox stress, protein nitration and deoxyribonucleic acid damage are induced. In SC, release of NO is usually increased, while asymmetric dimethylarginine, a competitive NO inhibitor, is consistently decreased. [21] It has been postulated that a catecholamine "storm", followed by $\beta 2$-adrenoreceptor coupling in the detriment of $\beta 1$-adrenoreceptors could be a mechanistic pathway in this disease. [22] This work has suggested that biased post-receptor signalling, leading to predominantly Gi-based stimulation in response to large concentrations of catecholamines, contributes towards the peri-apical negative inotropic changes typically seen in SC.This assertion was supported by the finding that blockade of Gi-based signalling by pertussis toxin prevented such changes. On the other hand administration of beta-adrenoceptor antagonists caused immediate death in this model, suggesting that this form of acute treatment should not be contemplated. It is also important to reiterate that the distribution of betaadrenergic receptors has never been explored in a human heart, therefore firm translation to the human condition remains a limitation.

Although this has only been demonstrated in an experimental model and not replicated in the human disease, the context of nitrosative stress could further explain the activation of nitric oxidase synthase, hence the increased NO production. Experimental data showed accumulation of 3-NT and PAR in the myocardium, most 
pronouncedly in the apical region, and these latter findings have been replicated in a human post-mortem series. [23] Moreover, SC patients demonstrate an aberrant response to NO, both in an acute setting and at a 3 months follow-up. The NO overresponsiveness has also been positively correlated to the severity of the disease. [21] Sodium nitroprusside (SNP), an index of platelet NO signaling, was linked to the peak NT-pro BNP serum concentration, suggesting the inflammatory component that NO might play a role in the illness. Interestingly, while the extent of the NO pathway up-regulation is related to the initial myocardial damage, it has also been shown to be a predictor of rapid recovery. Hydrogen sulphide (H2S) potentiates NO effects by further activation of the soluble guanyl cyclase. Additionally, $\mathrm{H} 2 \mathrm{~S}$ limits nitrosative stress via degradation of cysteine and glutathione. In an experimental rodent model, the concentration of $\mathrm{H} 2 \mathrm{~S}$ was found to be decreased, while administration of exogenous H2S had a beneficial effect by limiting the myocardial oxidative injury. [24] Another biochemical process noticed in SC is glycocalyx shedding. This is a marker of endothelial dysfunction, as well as the shear stress possibly encountered in the pathology. However, the only microvascular abnormality confirmed focuses solely on the coronary arteries. [25] The sequence of events that generates this process remains still unknown. It could be that the glycocalyx shedding is a primary phenomenon, as a result of catecholamine release and subsequent neutrophil burst. In this scenario, the myocardial inflammation could be a result of the vascular damage. Conversely, it might be a secondary alteration stimulated via BNPmediated inflammation. Quantitively, this process can be measured by Syndecan-1 (SD-1) in plasma, the most prevalent proteoglycan. Acutely SC patients have presented with a similar SD-1 level to AMI, significantly increased in comparison to controls, which normalised after three months. [26] Interestingly, the shedding of 
glycocalyx could perhaps explain the hypotension often co-occurring in SC patients, especially as the degree of hypotension is not correlated to the lowered LVEF level. Likewise, matrix metallo-proteinases (MMP's) drastically influence the release of glycocalyx. Recently, it has been demonstrated that an atypical MMP profile is present in SC patients in that collagenase-subtypes of MMPs were largely decreased: MMP1 by $40 \%$ and MMP8 by $60 \%$. [27] This does not only suggest a tendency to preserve collagen extracellularly, and thus a pro-fibrotic state, but also confirms an increase shedding of SD-1. [23] As a result of the oxidative stressinduced DNA damage, Poly [ADP-ribose] polymerase 1 (PARP-1, a first responder to DNA damage, facilitating DNA repair pathway choice) is activated. [28] Consequently, the energy impairment and lowered phosphocreatine levels could be explained. Moreover, the peroxynitrite anion stimulates the expression of $\alpha$-arrestin thioredoxin-interacting protein (TXNIP), a proinflammatory mediator involved in protein ubiquitination and endocytosis. TXNIP levels were analysed in a rat model and have been found elevated by 2.5 fold in SC-induced rodents compared to controls, with a significant apex-base gradient. TXNIP is associated with a turbulent blood flow, while also reinforcing the importance of shear stress in the disease. An accentuating factor of the abrupt increase of TXNIP, following the ISO administration, could be defined by the limitation of NO's protective effect. [23]

Despite systemic inflammation in SC only receiving attention in the recent years, the first indication of its importance was signaled in a case report by Sato in 2005. Further associations were then raised between the cardiomyopathy and microscopic polyangiitis, a type of inflammatory vasculitis. [29] In the case presented antiinflammatory therapy (methylprednisolone) was administered with remarkable results noticed, particularly in limiting the cardiac damage. Clearly the treatment would have 
suppressed the production of pro-inflammatory cytokines which have since been linked to the magnitude of the disease. This case report is yet equivocal in terms of management of SC patients, while randomised controlled trials have not been carried out. While many therapeutic targets have been postulated, the development of a precise treatment strategy remains premature until a better understanding of the pathophysiological processes is demonstrated.

Recently various cytokines have been evaluated for prognostic purposes. The research efforts have mainly focused on IL-6 (pro-inflammatory marker) and IL-10 (anti-inflammatory marker). IL-6 has proven to be significantly increased in AMI patients and has been now correlated with the rate of adverse effects in SC. The cytokine hypothesis has proposed that IL-6 induces muscle wasting and prompts heart failure. [30] IL-10 has been associated with negative cardiac remodeling. If both IL- 6 and IL-10 are raised upon admission, the risk of adverse effects has been estimated to $67 \%$, whereas if neither show a substantial increase the risk is lowered to $14 \%$. [31] IL-6 and IL-10 have also illustrated a positive correlation with CRP levels. Additionally, several other inflammatory markers have been found elevated in SC patients, respectively: IL-7 (interestingly, even at higher levels compared with AMI patients; this cytokine has been linked to the development of atherosclerotic plaques), growth regulated protein and chemokine ligand 1 (chemoattractant for neutrophils). [32]

Growth differentiation factor-15 (GDF-15) is a cytokine belonging to transforming growth factor $\beta$ family. It was shown to be released during stress-induced damage by cardiomyocytes and vascular smooth muscle cells and has been used for risk stratification in acute coronary syndrome patients. Stiermaier et al has illustrated that SC patients present with significantly increased GDF-15 compared to STEMI 
controls. Moreover, biventricular ballooning was a predictor factor for an elevated GDF-15. [33]

The cytokine surge and oedema also prompt the release of carbohydrate-antigen125 (CA-125) from pericardial cells. It has been positively correlated with NT-proBNP and is inversely proportional to the LVEF upon admission. CA-125 has been used as both a short-term and long-term prognostic tool for adverse outcomes and length of hospitalisation period. [34]

The innate immune system activation is also highlighted via the changes in white blood cell count. Both total white cell and neutrophil count were significantly elevated acutely ( $p<0.001$ compared with controls). Furthermore, monocyte subpopulations during the acute episode have shown notable alterations in a recently published study of our research group [8]. While the total monocyte count has shown no significant difference compared to controls, in the 0-3 days period after admission, a significant increase in the number of classical monocytes (CD14++CD16-, Mon1) to the detriment of intermediate (CD14++CD16+, Mon2) and non-classical (CD14+CD16++, Mon3) monocytes has been recorded. [8] Mon 1 are recruited in the acute inflammatory phase, accumulating in the injured areas of the myocardium and causing further inflammation by degrading proteins. It can be further hypothesised that the macrophage activity detected is a result of migration of Mon1 cells into the myocardium, rather than a proliferative effect of the already existent tissue resident macrophages. Moreover, Mon2 concentration remains decreased for a sustained period of time, in opposition to the process taking place in acute myocardial infraction: A change towards the Mon3 monocytes is noticed in the weeks following an $\mathrm{MI}$, thus limiting the pro-healing inflammatory processes. The sustained low concentration of Mon2 could therefore explain the unresolved 
myocardial inflammation in SC. The consistent increase over a period of 5 months in IL-8 and chemokine (C-X-C motif) ligand 1 (CXCL1), has been related to monocyte adhesion, as well as macrophage infiltration in the myocardium. In the TERRIFIC trial, in the acute setting, the mean concentration of IL-8 was $45.5 \pm 8.62(n=55)$ and increased over a period of 5 months to $61.9 \pm 10.28(n=48)$. The value reached clinical significance $(p=0.009)$ compared to matched control subjects $21.7 \pm 10.86(n=$ 51) at the 5 month follow-up. [8] Even more interestingly, the HEROIC trial analysed the serum cytokine profile at a median time of 20 months from the onset of the acute episode, thus analysing the longer-term inflammatory profile of the disease. An increased trend in the expression of CXCL1 in the patients with prior SC was again noticed compared to controls $(p=0.08)$. This finding further describes the specific clinical phenotype of SC in the recovery stage, respectively heart failure that presents in conjuncture with a low-grade, cytokine-mediated, systemic inflammatory state. [35]

Other studies have also revealed increased activation in the pro-thrombotic pathways, in SC patients compared to healthy controls, while the results were comparable to acute myocardial infraction (AMI) patients. This explains the relatively high (9.9\%) per patient-year risk of cardiac and cerebrovascular events. [36] Fitzgibbons et al tested 1,310 serum proteins in patients suffering from SC in comparison to AMI patients and controls. The upregulation of proteins was noticed mainly in the coagulation pathway and complement cascade. Only 6 proteins have been expressed differently solely in SC versus controls: complement 3 , factor $\mathrm{V}$, Follistatin Like 3 (increases adhesion of blood cells to fibronectin; inhibits TGF- $\beta$ signaling), Netrin-1 (upregulates endothelial NO production), PLAT and THBS2 
(matrix metalloproteinases secretion). [37] These proteins could become alternative cardiac biomarkers specific to SC.

There are several other biomarkers which have been proposed for the early detection of SC. First off, Micro-RNAs have also been studied in regard to their effectiveness as possible cardiac biomarkers. A signature profile of micro-RNA has been created for SC, respectively: increased miR1, miR16, miR26a and miR133a. [38] MiR1 and MiR133a are indicators of cardiac damage, whereas both miR16 and miR26a have been linked to stress and reduced cardiac contractility. Moreover, miR$371 \mathrm{a}-5 \mathrm{p}$ has been shown to have a slight correlation to the disease. It controls the regulation of Bcl2 associated anthogene 3 (BAG3). [39] BAG3 orchestrates the chaperone-assisted autophagy pathway, inhibiting apoptotic mechanisms via removal of faulty proteins. At least a mutation of this gene is present in $72.9 \%$ of SC patients, compared to $46.9 \%$ in the control population. However, the presented study only compared SC patients with healthy female controls and did not present other cardiac conditions in comparison. BAG3 has recently emerged as a key myocytic homeostatic control, being mutated in a wide variety of heart failure complexes. [40] Therefore the specificity of BAG3 and miR-371a-5p is limiting the clinical application of the results. Mi1-25a-5p was seen in unusual quantities in the plasma, suggesting a surge in endothelin-1. However, it is yet to be elucidated whether the microvascular impairment is an independent phenomenon, or a result of the extravascular obstruction caused by oedema. [37] Importantly, the results on MicroRNAs and genetic profiling currently do not have enough supporting information to be considered for their diagnostic utility. Secondly, serum copeptin was also analysed as a possible cardiac biomarker. As a vasopressin prohormone, which is released as result of a stressor event, it has been found to be highly elevated in SC. 
[41] This has only been reported in an isolated incident and therefore needs a more rigorous study design to reach a conclusion concerning its efficiency.

\section{DRAWING PARALLELS WITH OTHER CARDIAC INFLAMMATORY CONDITIONS}

While SC has been reported in association to a wide range of other diseases and comorbidities, a great density of cases has focused on the correlation between it and inflammation-mediated conditions, such as: septicemia, anaphylaxis, Kounis syndrome, hemophagocytic lymphohistiocytosis. This was expected given that mononuclear cells appear to play a vital role in the development of the illness.

Interestingly, sepsis is a major triggering factor for SC, some reports identifying it as the single most important contributor. $[42,43]$ The mechanisms that induce SC in this scenario could rely on cytokine toxicity, particularly Tumour necrosis factor- $\alpha$ and IL-1 $1 \beta$, impeding the cardiac contractility. Theoretically, anaphylactoid reaction could induce SC via similar mechanisms to sepsis. Anaphylaxis in the setting of SC is particularly challenging, since exogenous catecholamines could further worsen the cardiac dysfunction. Several case reports have associated hemophagocytic lymphohistiocytosis (HLH) with SC. [44, 45] HLH is described by multi-organ damage as a result of an acute systemic inflammation. In $\mathrm{HLH}$, the activated macrophages and histocytes phagocytise other blood cells. HLH causes organ dysfunction mainly via cytokine storms. Another disease SC has been compared to is Kounis syndrome (or allergic myocardial infraction). Type I is the most common form of the disease, resulting in coronary spasm as a consequence of an exposure to an allergen despite otherwise unobstructed coronary arteries. Since both diseases are prompted by similar inflammatory mediators, cases have been reported where the illnesses 
appeared in conjunction. [46] This correlation could dictate a direction for future research, perhaps in establishing the relation between inflammatory mediators and SC.

\section{FUTURE RESEARCH AND CONTROVERSIES}

Current advancements reveal more dilemmas concerning the pathological processes underlying SC. It is clear that inflammation is an integral part of the disease, but the cause-effect relationship remains unknown. It could be that the inflammation in this disease is a secondary phenomenon, perhaps precipitated by autonomic imbalance/overdrive or micro-vascular abnormalities, or conversely the inflammatory process being causative in the course of SC, driving the microcirculatory abnormalities through a mechanical, compressing effect of oedema for example.

It is imperative to continue probing on the mechanism of the disease. The hypotheses formulated so far, respectively: the catecholamine surge, sympathetic overdrive, multi-vessel epicardial spasms, microvascular dysfunction, and inflammation, have failed to fully explain the evolution of this complex syndrome. The postulated proposals do not reciprocally exclude each other, while the typical phenotype could be a result of their synergistic action. Understanding the mechanistic pathways is the cornerstone to elucidating the most effective therapeutic actions.

As the pathophysiology of SC remains largely elusive, no international guidelines in terms of treatment have reached consensus. Given the current knowledge of the disease, it is safe to assume that administration of exogenous catecholamines should be avoided, at least until further clarification. Retrospective studies have indicated that beta-blockers are not effective in the reduction of in-hospital mortality 
or recurrence rate. Anti-inflammatory agents have been suggested to act as future therapeutic targets. Nonetheless, there is still a lot to be investigated before randomised control trials with a correspondent evidence-based therapeutic management could be performed. Otherwise, in the case of complications (cardiogenic shock/ cardiac arrhythmias) the standard pharmacological or mechanical support is usually recommended.

Furthermore, there is an urgent need for more pertinent cardiac biomarkers, ideally to distinguish it rapidly as clinical phenocopy of myocardial infarction. Several markers of inflammation, ventricular stretch and gene expression have shown a significant correlation to the disease (BNP: troponin/myoglobin, microRNA, cytokines, copeptin). Despite constant progress, search, as to which biomarker should be used, continues.

Table 1- The results of T2-weighted images in relation to myocardial oedema in SC

\begin{tabular}{|c|c|c|c|}
\hline Study & $\begin{array}{l}\text { Myocardial } \\
\text { oedema (acutely) }\end{array}$ & LGE detected & Other findings \\
\hline $\begin{array}{l}\text { 2009, Abdel-Aty } \\
\text { et al [47] }\end{array}$ & $100 \%(7 / 7)$ & $-\quad *$ & $\begin{array}{l}\text { Minute foci of } \\
\text { intramural LE } \\
(14.29 \%)\end{array}$ \\
\hline \multirow[t]{2}{*}{$\begin{array}{l}\text { 2009, Eitel et al } \\
\text { [48] }\end{array}$} & $42.3 \%(11 / 26)$ & $-\quad * *$ & $\begin{array}{l}\text { Pericardial } \\
\text { effusion (57.7\%) }\end{array}$ \\
\hline & $\begin{array}{l}69 \%(18 / 26)- \\
\text { elevated T2 ratio }\end{array}$ & & \\
\hline
\end{tabular}




\begin{tabular}{llll}
\hline $\begin{array}{l}\text { 2009, Rolf et al } \\
\text { [49] }\end{array}$ & $100 \%(15 / 15)$ & $33.3 \%(5 / 15)$ & - \\
\hline 2011, Eitel et al & $81 \%(162 / 199)$ & $22 \%(9 / 199)$ & $\begin{array}{l}\text { Pericardial } \\
\text { effusion }(44 \%) ;\end{array}$ \\
[50] & & & $\begin{array}{l}\text { Pleural effusion } \\
(33 \%)\end{array}$
\end{tabular}

\begin{tabular}{|c|c|c|c|}
\hline $\begin{array}{l}\text { 2012, Neil et al } \\
\text { [13] }\end{array}$ & $100 \%(32 / 32)$ & $3.13 \%(1 / 32)$ & $\begin{array}{l}\text { Correlation of } \\
\text { degree of } \\
\text { intracardiac } \\
\text { oedema to NT- } \\
\text { proBNP }\end{array}$ \\
\hline
\end{tabular}

2012, Nakamori $\quad 96 \%(22 / 23) \quad 22 \%(5 / 23)$

et al [51]

*LGE not attempted

**at the time LGE detection was an exclusion criterion for SC

Table 2- Concentration of IL- 6 at admission of patients suffering from SC

\begin{tabular}{llll}
\hline Research study & $\begin{array}{l}\text { Patients with SC } \\
\text { acutely } \\
\text { pg/mL } \\
\text { (number of } \\
\text { participants) }\end{array}$ & $\begin{array}{l}\text { MATCHED CONTROL } \\
\text { SUBJECTS }\end{array}$ & $\begin{array}{l}\text { P-VALUE } \\
\text { (COMPARED TO } \\
\text { CONTROLS) }\end{array}$ \\
\hline $\begin{array}{l}\text { 2019, C. Scally } \\
\text { et al [35] }\end{array}$ & $23 \pm 7(29)$ & $18 \pm 10$ & 0.7 \\
\hline $\begin{array}{l}\text { 2018, C. Scally } \\
\text { et al [8] }\end{array}$ & $23.1 \pm 4.54(55)$ & $6.5 \pm 5.83$ & $<0.001$ \\
\hline
\end{tabular}


2018, F. Santoro $12.37 \pm 17.48(32) \quad 25.42 \pm 27.71^{*} \quad 0.03$

et al [52]

2016, F. Santoro $41.25 \pm 136$ (56)

et al [31]

2011, R. Pirzer $\quad 2.1 \pm 2.6^{* *}(16) \quad 5.2 \pm 5.0^{*} \quad 0.021$

et al [32]

* Control subjects suffered from acute coronary syndrome

${ }^{* *}$ More elevated after the day of admission 


\section{REFERENCES}

[1] Sato H, TateishiH, Uchida T, Dote K, Ishihara M, Kodama K et al. Clinical aspect of myocardial injury: from ischemia to heart failure. Kagaku Hyoronsha. 1990:55-64. [2] Khalid N, Ahmad SA, Shlofmitz E, Chhabra L. Racial and gender disparities among patients with Takotsubo syndrome. Clinical Cardiology. 2019;42(1):19. [3] Scantlebury DC, Prasad A, Prasad A. Diagnosis of takotsubo cardiomyopathy Mayo Clinic criteria -. Circulation Journal. 2014;78(9):2129-2139 [4] Carita P, Fazio G, Novo G, Novo G. Takotsubo cardiomyopathy. E-journal Cardiol. Pract. 2010;8.

[5] Prasad A, Lerman A, Rihal CS. Apical ballooning syndrome (Tako-Tsubo or stress cardiomyopathy): a mimic of acute myocardial infarction. American Heart Journal. 2008;155(3):408-17.

[6] Neil CJ, Nguyen TH, Singh K, Raman B, Stansborough J, Dawson D, Frenneaux MP, Horowitz JD. Relation of delayed recovery of myocardial function after takotsubo cardiomyopathy to subsequent quality of life. The American journal of cardiology. $2015 ; 15 ; 115(8): 1085-9$.

[7] Singh K, Carson K, Shah R, Sawhney G, Singh B, Parsaik A et al. Meta-analysis of clinical correlates of acute mortality in takotsubo cardiomyopathy. The American journal of cardiology. 2014;113(8):1420-8.

[8] Scally C, Abbas H, Ahearn T, Srinivasan J, Mezincescu A, Rudd A et al. Myocardial and systemic inflammation in acute stress-induced (Takotsubo) cardiomyopathy. Circulation. 2019;139(13):1581-92.

[9] Perazzolo Marra M, Lima JA, lliceto S. MRI in acute myocardial infarction. European heart journal. 2011;32(3):284-93. 
[10] Kagiyama N, Okura H, Tamada T, Imai K, Yamada R, Kume T et al. Impact of right ventricular involvement on the prognosis of takotsubo cardiomyopathy. European Heart Journal-Cardiovascular Imaging. 2016;17(2):210-6.

[11] Nef HM, Möllmann H, Hilpert P, Troidl C, Voss S, Rolf A et al. Activated cell survival cascade protects cardiomyocytes from cell death in Tako - Tsubo cardiomyopathy. European journal of heart failure. 2009;11(8):758-64. [12] Aikawa Y, Noguchi T, Morita Y, Tateishi E, Kono A, Miura H, Komori Y, Asaumi Y, Fukuda T, Yasuda S. Clinical impact of native T1 mapping for detecting myocardial impairment in takotsubo cardiomyopathy. European Heart JournalCardiovascular Imaging. 2019;20(10):1147-55.

[13] Neil C, Nguyen TH, Kucia A, Crouch B, Sverdlov A, Chirkov Y et al. Slowly resolving global myocardial inflammation/oedema in Tako-Tsubo cardiomyopathy: evidence from T2-weighted cardiac MRI. Heart. 2012;98(17):1278-84.

[14] Scally C, Ahearn T, Rudd A, Neil CJ, Srivanasan J, Jagpal B et al. Right ventricular involvement and recovery after acute stress-induced (tako-tsubo) cardiomyopathy. The American journal of cardiology. 2016;117(5):775-80. [15] Nef HM, Möllmann H, Kostin S, Troidl C, Voss S, Weber M et al. Tako-Tsubo cardiomyopathy: intraindividual structural analysis in the acute phase and after functional recovery. European heart journal. 2007;28(20):2456-64.

[16] Surikow SY, Raman B, Licari J, Singh K, Nguyen TH, Horowitz JD. Evidence of nitrosative stress within hearts of patients dying of Tako-tsubo cardiomyopathy. International journal of cardiology. 2015;189:112-4.

[17] Wilson HM, Cheyne L, Brown PA, Kerr K, Hannah A, Srinivasan J et al. Characterization of the myocardial inflammatory response in acute stress-induced 
(Takotsubo) cardiomyopathy. JACC: Basic to Translational Science. 2018;3(6):76678.

[18] Madhavan M, Borlaug BA, Lerman A, Rihal CS, Prasad A. Stress hormone and circulating biomarker profile of apical ballooning syndrome (Takotsubo cardiomyopathy): insights into the clinical significance of B-type natriuretic peptide and troponin levels. Heart. 2009;95(17):1436-41.

[19] Fröhlich GM, Schoch B, Schmid F, Keller P, Sudano I, Lüscher TF, Noll G, Ruschitzka F, Enseleit F. Takotsubo cardiomyopathy has a unique cardiac biomarker profile: NT-proBNP/myoglobin and NT-proBNP/troponin T ratios for the differential diagnosis of acute coronary syndromes and stress induced cardiomyopathy. International journal of cardiology. 2012 Feb 9;154(3):328-32. [20] Nguyen TH, Neil CJ, Sverdlov AL, Mahadavan G, Chirkov YY, Kucia AM et al. $\mathrm{N}$-terminal pro-brain natriuretic protein levels in takotsubo cardiomyopathy. The American journal of cardiology. 2011 Nov 1;108(9):1316-21.

[21] Nguyen TH, Neil CJ, Sverdlov AL, Ngo DT, Chan WP, Heresztyn T et al. Enhanced NO signaling in patients with Takotsubo cardiomyopathy: short-term pain, long-term gain?. Cardiovascular drugs and therapy. 2013;27(6):541-7.

[22] Paur H, Wright PT, Sikkel MB, Tranter MH, Mansfield C, O'gara P et al. High Levels of Circulating Epinephrine Trigger Apical Cardiodepression in a $\beta 2-$ Adrenergic Receptor/Gi-Dependent Manner: A New Model of Takotsubo Cardiomyopathy. Circulation. 2012;126(6):697-706. [23] Surikow SY, Nguyen TH, Stafford I, Chapman M, Chacko S, Singh K et al. Nitrosative stress as a modulator of inflammatory change in a model of Takotsubo syndrome. JACC: Basic to Translational Science. 2018 Apr 30;3(2):213-26. 
[24] Zhang Z, Jin S, Teng X, Duan X, Chen Y, Wu Y. Hydrogen sulfide attenuates cardiac injury in takotsubo cardiomyopathy by alleviating oxidative stress. Nitric oxide. $2017 ; 67: 10-25$.

[25] Cecchi E, Parodi G, Giglioli C, Passantino S, Bandinelli B, Liotta AA et al.

Stress-induced hyperviscosity in the pathophysiology of takotsubo cardiomyopathy. The American journal of cardiology. 2013;111(10):1523-9.

[26] Nguyen TH, Liu S, Ong GJ, Stafford I, Frenneaux MP, Horowitz JD. Glycocalyx shedding is markedly increased during the acute phase of Takotsubo cardiomyopathy. International journal of cardiology. 2017;243:296-9. [27] Essa EM, Zile MR, Stroud RE, Rice A, Gumina RJ, Leier CV et al. Changes in plasma profiles of matrix metalloproteinases (MMPs) and tissue inhibitors of MMPs in stress-induced cardiomyopathy. Journal of cardiac failure. 2012;18(6):487-92. [28] Lipowsky HH, Lescanic A. The effect of doxycycline on shedding of the glycocalyx due to reactive oxygen species. Microvascular research. 2013;90:80-5. [29] Sato T, Hagiwara K, Nishikido A, Miyamoto S, Komiyama K, Matsuno H et al. Takotsubo (ampulla-shaped) cardiomyopathy associated with microscopic polyangiitis. Internal medicine. 2005;44(3):251-5.

[30] Seta Y, Shan K, Bozkurt B, Oral H, Mann DL. Basic mechanisms in heart failure: the cytokine hypothesis. Journal of cardiac failure. 1996;2(3):243-9.

[31] Santoro F, Tarantino N, Ferraretti A, leva R, Musaico F, Guastafierro F et al. Serum interleukin 6 and 10 levels in Takotsubo cardiomyopathy: increased admission levels may predict adverse events at follow-up. Atherosclerosis. 2016;254:28-34. 
[32] Pirzer R, Elmas E, Haghi D, Lippert C, Kralev S, Lang S et al. Platelet and monocyte activity markers and mediators of inflammation in Takotsubo cardiomyopathy. Heart and vessels. 2012;27(2):186-92.

[33] Stiermaier T, Adams V, Just M, Blazek S, Desch S, Schuler G et al. Growth differentiation factor-15 in Takotsubo cardiomyopathy: diagnostic and prognostic value. International journal of cardiology. 2014;173(3):424-9.

[34] Santoro F, Ferraretti A, Musaico F, Di Martino L, Tarantino N, leva R et al. Carbohydrate-antigen-125 levels predict hospital stay duration and adverse events at long-term follow-up in Takotsubo cardiomyopathy. Internal and emergency medicine. 2016;11(5):687-94.

[35] Scally C, Rudd A, Mezincescu A, Wilson H, Srivanasan J, Horgan G et al. Persistent long-term structural, functional, and metabolic changes after stressinduced (Takotsubo) cardiomyopathy. Circulation. 2018;137(10):1039-48. [36] Templin C, Ghadri JR, Diekmann J, Napp LC, Bataiosu DR, Jaguszewski M et al. Clinical features and outcomes of Takotsubo (stress) cardiomyopathy. New England Journal of Medicine. 2015;373(10):929-38.

[37] Fitzgibbons TP, Edwards YJ, Shaw P, Iskandar A, Ahmed M, Bote J et al. Activation of Inflammatory and Pro-Thrombotic Pathways in acute stress cardiomyopathy. Frontiers in cardiovascular medicine. 2017;4:49. [38] Jaguszewsski M, Osipova J, Ghadri JR, Napp LC, Widera C, Franke J et al. A signature of circulating microRNAs differentiates takotsubo cardiomyopathy from acute myocardial infarction. European heart journal. 2014;35(15):999-1006. [39] d'Avenia M, Citro R, De Marco M, Veronese A, Rosati A, Visone R et al. A novel miR-371a-5p-mediated pathway, leading to BAG3 upregulation in cardiomyocytes in 
response to epinephrine, is lost in Takotsubo cardiomyopathy. Cell death \& disease. 2015;6(10):e1948.

[40] Knezevic T, Myers VD, Gordon J, Tilley DG, Sharp TE, Wang J et al. BAG3: a new player in the heart failure paradigm. Heart failure reviews. 2015;20(4):423-34. [41] Wu AH, Tabas JA, Vollert JO, Lauterbach T, Mockel M. Results of novel cardiac biomarkers in Tako-Tsubo cardiomyopathy. International journal of cardiology. 2012;159(1):53-5.

[42] Cappelletti S, Ciallella C, Aromatario M, Ashrafian H, Harding S, Athanasiou T. Takotsubo cardiomyopathy and Sepsis: a systematic review. Angiology. 2017;68(4):288-303.

[43] Park JH, Kang SJ, Song JK, Kim HK, Lim CM, Kang DH et al. Left ventricular apical ballooning due to severe physical stress in patients admitted to the medical ICU. Chest. 2005;128(1):296-302.

[44] Otillio JK, Harris JK, Tuuri R. A 6-year-old girl with undiagnosed hemophagocytic lymphohistiocytosis and takotsubo cardiomyopathy: a case report and review of the literature. Pediatric emergency care. 2014;30(8):561-5.

[45] Koh S, Wu C, Cypro A, Castellanos L. Hemophagocytic lymphohistiocytosis associated Takotsubo cardiomyopathy and survival after ventricular fibrillation arrest. Journal of the American College of Cardiology. 2019;73(9 Supplement 1):2599. [46] Yanagawa Y, Nishi K, Tomiharu N, Kawaguchi T. A case of takotsubo cardiomyopathy associated with Kounis syndrome. International journal of cardiology. 2009;132(2):e65-7.

[47] Abdel-Aty H, Cocker M, Friedrich MG. Myocardial edema is a feature of TakoTsubo cardiomyopathy and is related to the severity of systolic dysfunction: insights 
from T2-weighted cardiovascular magnetic resonance. International journal of cardiology. 2009;132(2):291-3.

[48] Eitel I, Lücke C, Grothoff M, Sareban M, Schuler G, Thiele H et al. Inflammation in takotsubo cardiomyopathy: insights from cardiovascular magnetic resonance imaging. European radiology. 2010;20(2):422-31.

[49] Rolf A, Nef HM, Möllmann H, Troidl C, Voss S, Conradi G et al. Immunohistological basis of the late gadolinium enhancement phenomenon in takotsubo cardiomyopathy. European heart journal. 2009;30(13):1635-42. [50] Eitel I, von Knobelsdorff-Brenkenhoff F, Bernhardt P, Carbone I, Muellerleile K, Aldrovandi A et al. Clinical characteristics and cardiovascular magnetic resonance findings in stress (takotsubo) cardiomyopathy. Jama. 2011;306(3):277-86.

[51] Nakamori S, Matsuoka K, Onishi K, Kurita T, Ichikawa Y, Nakajima H et al. Prevalence and signal characteristics of late gadolinium enhancement on contrastenhanced magnetic resonance imaging in patients with takotsubo cardiomyopathy. Circulation Journal. 2012;76(4):914-21.

[52] Santoro F, Costantino MD, Guastafierro F, Triggiani G, Ferraretti A, Tarantino N et al. Inflammatory patterns in Takotsubo cardiomyopathy and acute coronary syndrome: A propensity score matched analysis. Atherosclerosis. 2018;274:157-61. 